\title{
A massive lipoma in a patient receiving chlorpropamide therapy
}

\author{
Henry A. Garfinkel \\ M.B., B.S., L.R.C.P., M.R.C.S. \\ St Mary's Hospital, London, W.2
}

LipomatA are common benign tumours most frequently situated in the subcutaneous tissues. They may be single or multiple. Although serious complications are exceedingly rare, haemorrhage, torsion and malignant change can very infrequently occur.

The patient to be described presented with a huge lipoma over his upper back. This and a speculative theory of causation are discussed.

\section{Clinical and pathological report}

A 60-year-old Pakistani had been aware of a tumour of increasing size for about 16 years. Initially, the lump was very small and it had only slowly increased in size for several years. For about the last 5 years it grew rapidly to reach its present massive dimensions, approximating to the size of a Rugby football. The lump originated by a relatively narrow stalk from his upper back. It was freely mobile and its surface was ulcerated in parts, probably due to local pressure. Large veins were clearly visible in the overlying skin (Fig. 1). Some skin tethering was present and the tumour surface felt lobulated.

Also for about 16 years, the patient had been thought to have diabetes mellitus. There was no clinical or laboratory evidence for this but he had been taking chlorpropamide, an oral hypoglycaemic agent, for several years-probably eight.

His health was otherwise satisfactory.

Chest radiography showed an immense posterior soft tissue swelling but no other abnormality (Fig. 2). A standard glucose tolerance curve was unequivocally normal after a few days without chlorpropamide (blood glucose $67 \mathrm{mg} / 100 \mathrm{ml}$, fasting, and then $102,123,91$ and $72 \mathrm{mg} / 100 \mathrm{ml}$ at $\frac{1}{2} \mathrm{hr}$ intervals). His protein bound iodine level was $5 \cdot 7$ $\mu \mathrm{g} / 100 \mathrm{ml}$.

\section{Management}

The patient was anaesthetized, his tumour immobilized with a hook and the lump, together with a small intradermal fatty tumour, was removed. Large skin flaps were retained and the wound sutured.

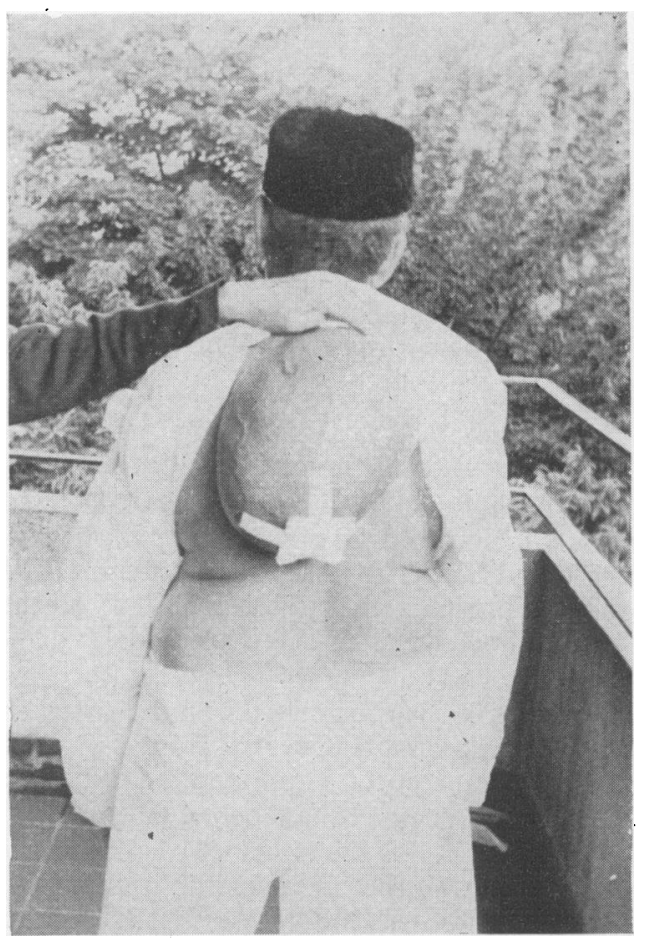

Fig. 1. Massive lipoma-note enlarged veins and dressing over ulcerated area.

Apart from a mild post-operative pyrexia, his recovery was uneventful.

The lump measured $30.0 \mathrm{~cm} \times 24.0 \mathrm{~cm} \times$ up to $20 \mathrm{~cm}$ thick and weighed $5 \cdot 1 \mathrm{~kg}$. Histology revealed extensive areas of fat necrosis in a massive lipoma.

\section{Discussion}

The aetiology of lipomata is unknown. Genetic, endocrine and other conditions are sometimes also present in patients with lipomata (Osment, 1968) but none of these were operative in this patient. Early in this century it was thought that the fat of lipomata was strictly comparable with adipose tissue at other 


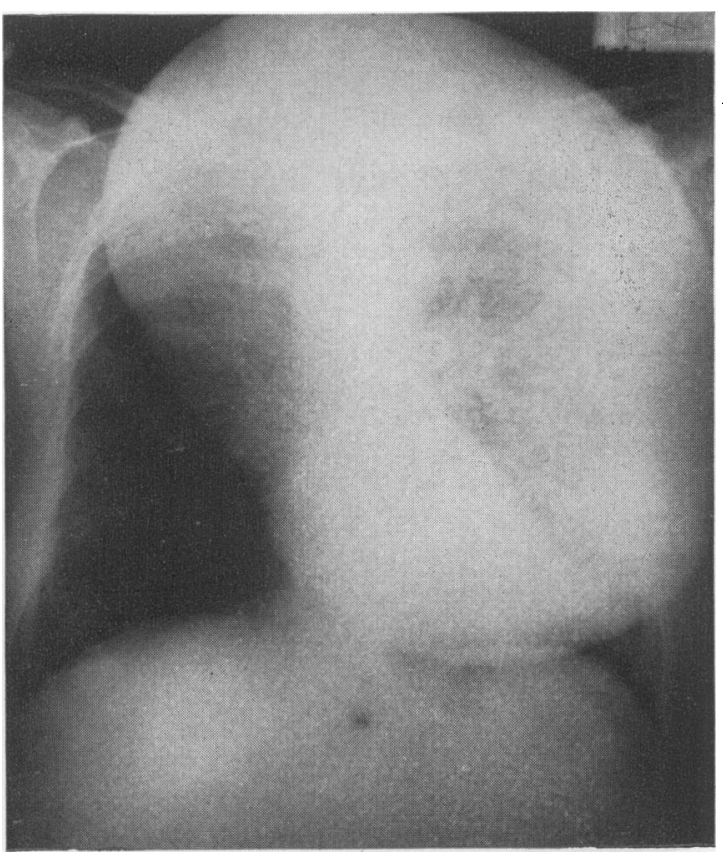

FIG. 2. Chest X-ray showing huge soft tissue swelling.

sites (Wells, 1912) but more recently the metabolic activity of lipoma fat was shown to differ from normal subcutaneous fat: 'fatty acid precursors are incorporated more rapidly into lipoma fat while lipoprotein lipase activity is diminished' (Osment, 1968). Tranquada (1966) investigated a diabetic patient who had subcutaneous lipomata at sites of insulin injection. He incubated adipose tissue from one of these lipomata and also from surrounding nearby subcutaneous fat and found the former to be more efficient in converting labelled glucose to fatty acid, especially under the influence of added insulin. Glucose seems to be the most important factor concerned in lipolysis or lipogenesis. If a starving animal is fed with glucose, or a diabetic animal is treated with insulin and glucose, lipogenesis is favoured. It is interesting that a patient with a lipoma who is wasting from serious disease may find that his lipoma is increasing in size (Wells, 1912).
Although many compounds were used to treat diabetes prior to the discovery of insulin in 1922, it is $\varrho$ only comparatively recently that moderately safe and $z$ efficient oral drugs have been used extensively for $\stackrel{\varnothing}{\varrho}$ clinical purposes. Chlorpropamide, a sulphonylurea, $\subseteq$ was introduced in 1958 and has been used in- $\overrightarrow{\vec{F}}$ creasingly since then.

Sulphonylureas were long thought to act solely by stimulating the pancreatic beta cells to release more $\frac{\bar{c}}{\mathrm{c}}$ insulin into the circulation (Loubatières, 1957). $\vec{\nabla}$ Recent work suggests that sulphonylureas also suppress glucagon production by the pancreatic is alpha cells (Samols, Tyler \& Mialhe, 1969) and $\vec{\circ}$ potentiate the peripheral action of insulin (Madsen, 1967).

It may be that the patient under discussion had a small lipoma which eventually grew to its enormous size because of the action of the chlorpropamide hehad unnecessarily been taking for many years, in association with a normal diet. The lipoma may have more avidly incorporated glucose into its meta- + bolic pathways than the rest of his body because of $\vec{w}$ the presumed increase in his serum insulin levels. 0 Most of the increase in size had, in fact, occurred i⿱⺈ recent years whilst he was on this drug.

\section{Acknowledgments}

I wish to thank Mr D. Mulvaney, who performed operation, Dr A. D. Thomson for the pathological detadis of the lipoma and Dr E. Besterman who, in addition to $\vec{\theta}$ above, gave me valuable advice in preparing this paper.

\section{References}

LOUBATIÈRES, A. (1957) The mechanism of action of the hypoglycaemic sulphonamides: a concept based on in-o vestigations in animals and man. Diabetes, 6, 408.

MADSEN, J. (1967) Extrapancreatic and intrapancreatic action of antidiabetic sulphonylureas. A review. Acta medica Scandinavica, 476, 109.

OSMENT, L.S. (1968) Cutaneous lipomas and lipomatosis. Surgery, Gynaecology and Obstetrics, 126, 129.

Samols, E., Tyler, J.M. \& Mialhe, P. (1969) Suppressionof pancreatic glucagon release by the hypoglycaemic금 sulphonylureas. Lancet, i, 174.

Tranquada, R.E. (1966) Subcutaneous lipomas at sites of insulin infection. Diabetes, 15, 807.

Wells, H.G. (1912) The fat metabolism of lipomas. Archives of Internal Medicine, 10, 297. 